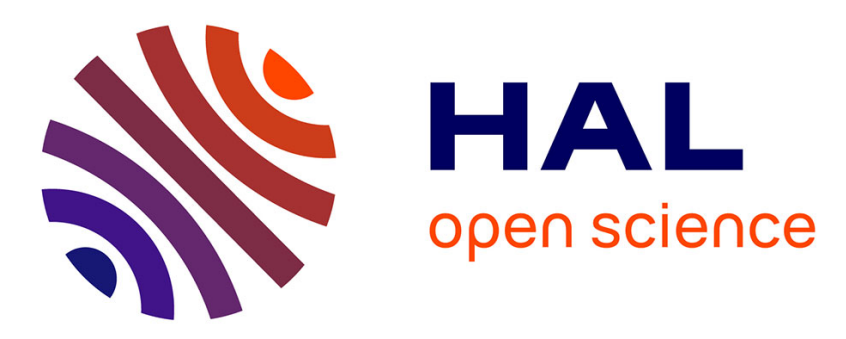

\title{
Surface Plasmon Retardation in Graphene Bilayer
}

Konstantin Batrakov, Vasil Saroka

\section{To cite this version:}

Konstantin Batrakov, Vasil Saroka. Surface Plasmon Retardation in Graphene Bilayer. Nanomaterials Imaging Techniques, Surface Studies, and Applications Selected Proceedings of the FP7 International Summer School Nanotechnology: From Fundamental Research to Innovations, August 26-September 2, 2012, Bukovel, Ukraine , Springer Verlag (Germany), pp.103-115, 2013, 10.1007/978-1-4614-76757_9. hal-01707618

\section{HAL Id: hal-01707618 https://hal.science/hal-01707618}

Submitted on 12 Feb 2018

HAL is a multi-disciplinary open access archive for the deposit and dissemination of scientific research documents, whether they are published or not. The documents may come from teaching and research institutions in France or abroad, or from public or private research centers.
L'archive ouverte pluridisciplinaire HAL, est destinée au dépôt et à la diffusion de documents scientifiques de niveau recherche, publiés ou non, émanant des établissements d'enseignement et de recherche français ou étrangers, des laboratoires publics ou privés. 


\title{
Surface plasmon retardation in graphene bilayer
}

\author{
Konstantin Batrakov and Vasil Saroka
}

\begin{abstract}
Surface plasmons can be used for generation of radiation by Čerenkov mechanism in carbor nanotubes. However, slowing down of the plasmon phase speed is not enough for the synchronization with a non-relativistic electron beam. Using the density matrix formalism and the tight-binding approximation, we developed the method of obtaining the dispersion equation for plasmons in $n$-layer graphene systems. It was found that a graphene single layer can reduce the surface plasmon phase speed by 3-6 times. Reduction up to the Fermi velocity of $\pi$-electrons is achieved in spatially separated graphene bilayer. Thus, graphene bilayer seems to be suitable material for realization of the Cerenkov-type emitters in nanoscale.
\end{abstract}

\section{Introduction}

In recent years, an enormous interest has been surrounding the field of plasmonics, because of the variety of tremendously exciting and novel phenomena it could enable. Plasmonics seems to be the only viable path toward realization of nanophotonics: control of light at scales substantially smaller than the wavelength [1]. Accessing subwavelength optical length scales introduces the prospect of compact optical devices with new functionalities by enhancing inherently weak physical processes, such as fluorescence and Raman scattering of single molecules [2] and nonlinear phenomena [3]. An optical source that couples electronic transitions directly to strongly localized optical modes is highly desirable because it would avoid the

Konstantin Batrakov

Institute for Nuclear Problems, Belarusian State University, Bobruyskaya 11, 220030 Minsk,

Belarus e-mail: kgbatrakov@gmail.com

Vasil Saroka

Institute for Nuclear Problems, Belarusian State University, Bobruyskaya 11, 220030 Minsk, Belarus, e-mail: 40.ovasil@gmail.com 
limitations of delivering light from a macroscopic external source to the nanometre scale, such as low coupling efficiency and difficulties in accessing individual optical modes [4]. On the other hand, plasmonics is a crucial ingredient in the reverse process, namely the generation of coherent radiation [5].

The one of mechanisms of the generation was suggested in the work [6]. The idea utilizes the effect of wave slowing down in waveguides $[7,8,9]$ and uses the analogy between carbon nanotubes(CNT) and traveling-wave tubes. Three basic properties of carbon nanotubes: the strong slowing down of surface electromagnetic waves [10], the ballisticity of the electron motion over typical CNT length [11, 12], and the extremely high electron current density reachable in CNTs [13], allow proposition of them as the candidates for the development of the nanoscale Čerenkov-type emitters. Theoretical treatment showed the possibility of such devices operation. But requirements that are put forward to the parameters of the devices operation are very strict, such as electron current density $\approx 10^{9} \mathrm{~A} / \mathrm{cm}^{2}$, nanotube length $\approx 10 \mu \mathrm{m}$ and so on. The main problem is that the retardation is not strong enough in nanotubes both single-wall and multi-wall. In fact the retardation of surface modes is higher for multi wall nanotubes [14] and it can be interpreted in terms of the subtraction of plasmon oscillations frequencies of adjacent layers [14]. But in nanotubes the frequencies of plasmon oscillations differ from layer to layer because of the changes of layers radii. This obstacle can be eliminated in planar systems. This greatly motivates us to explore the plasmon dispersion and the plasmon phase speed in a newly available material with unique properties: graphene and graphene multilayer.

The paper is organized as follows. In Sect.(2) a self-consistent problem is stated and the basic equation for the electromagnetic wave propagating in the $n$-layer graphene electron plasma is derived. A solution of this equation describing confined to graphene surface electromagnetic wave is presented in Sect.(3). The dispersion equations describing the slowing effect in graphene (graphene bilayer) are it derived and discussed in Sect.(4). Analysis and concluding remarks are given in Sect.(5).

\section{Self-consistent problem basic equation}

Let us consider the electromagnetic wave propagating and interacting with electron system in an $n$-layer graphene. To describe such system Maxwell's equations for electromagnetic wave and quantum mechanical equations should be used. The former can be reduced to the wave equation for a scalar potential:

$$
\left(\frac{\partial^{2}}{c^{2} \partial t^{2}}-\Delta\right) \Phi(\mathbf{r}, t)=4 \pi \rho(\mathbf{r}, t),
$$

where $\Phi(\mathbf{r}, t)$ is the scalar potential and $\rho(\mathbf{r}, t)$ is an electron charge density. Equation (1) should be supplemented by equation for the electron charge density. Manybody formalism is very useful for this purpose. Within the framework of this formalism the electron charge density take the following form: 


$$
\rho(\mathbf{r}, t)=e\langle 0|\widehat{\rho}| 0\rangle
$$

where $e$ is the elementary charge, $|0\rangle$ is the ground state of the system under consideration, $\widehat{\rho}$ is referred to as the density operator, having the following form:

$$
\widehat{\rho}=\widehat{\Psi}^{\dagger} \widehat{\Psi}
$$

where $\widehat{\Psi}$ is referred to the $\widehat{\Psi}$-operator or the secondary quantized electron wave function, and symbol ${ }^{\dagger}$ denotes Hermitian conjugation. The function is a decomposition over a full set of the possible stationary states of the system:

$$
\widehat{\Psi}=\sum_{\mathbf{k} s} \psi_{s}(\mathbf{r}, \mathbf{k}) \widehat{b}_{\mathbf{k} s}(t)
$$

where summation is performed over the stationary states with a quasimomentum $\mathbf{k}$ as well as over all energy bands numbered with index $s, \psi_{s}(\mathbf{r}, \mathbf{k})$ is the wave function of the electron with the quasimomentum $\mathbf{k}$, in the $s$-th energy band, and $\widehat{b}_{\mathbf{k} s}(t)$ is the time dependent annihilation operator for the electron in the state corresponding to subscript indexes. The time evolution of the creation and annihilation operators is governed by the Heisenberg equation, which is an analogue of the Schrödinger one, and has the following form:

$$
\frac{\mathrm{d} \widehat{b}_{\mathbf{k} s}(t)}{\mathrm{d} t}=-\frac{1}{\mathrm{i} \hbar}\left[H, \widehat{b}_{\mathbf{k} s}(t)\right],
$$

where $H$ is the Hamiltonian that can be written in the form:

$$
\widehat{H}=\widehat{H}_{0}+\widehat{H}_{\text {int }}
$$

here $\widehat{H}_{0}$ is an operator of the energy of the $n$-layer graphene electron plasma, for the case when the electromagnetic wave is absent, and $\widehat{H}_{\text {int }}$ is the interaction Hamiltonian. Operator $\widehat{H}_{0}$ has the form:

$$
\widehat{H}_{0}=\sum_{\mathbf{k} s} E_{\mathbf{k} s} \widehat{b}_{\mathbf{k} s}^{\dagger}(t) \widehat{b}_{\mathbf{k} s}(t),
$$

where $E_{\mathbf{k} s}$ is the unperturbed energy of the electron having quasimomentum $\mathbf{k}$ in the $s$-th energy band, the product of the creation and annihilation operators in Eq.(7) is usually called occupation number operator. The interaction Hamiltonian can be written as:

$$
\widehat{H}_{\text {int }}=\sum_{\mathbf{k}_{1} s_{1}} \sum_{\mathbf{k}_{2} s_{2}} H_{\mathbf{k}_{1} s_{1}, \mathbf{k}_{2} s_{2}}^{\mathrm{int}}(t) \widehat{b}_{\mathbf{k}_{1} s_{1}}^{\dagger}(t) \widehat{b}_{\mathbf{k}_{2} s_{2}}(t),
$$

where $H_{\mathbf{k}_{1} s_{1}, \mathbf{k}_{2} s_{2}}^{\mathrm{int}}(t)$ is the matrix element, having the following form:

$$
H_{\mathbf{k}_{1} s_{1}, \mathbf{k}_{2} s_{2}}^{\mathrm{int}}(t)=e \int \Phi(\mathbf{r}, t) \psi_{s_{1}}^{*}\left(\mathbf{r}, \mathbf{k}_{1}\right) \psi_{s_{2}}\left(\mathbf{r}, \mathbf{k}_{2}\right) \mathrm{d} \mathbf{r},
$$


From the Eqs.(3-9), the following two systems describing dynamics of creation and annihilation operators can be obtained:

$$
\begin{aligned}
& \frac{\mathrm{d} \widehat{b}_{\mathbf{k} s}(t)}{\mathrm{d} t}=-\frac{\mathrm{i}}{\hbar}\left(E_{\mathbf{k} s} \widehat{b}_{\mathbf{k} s}(t)+\sum_{\mathbf{k}_{1} s_{1}} H_{\mathbf{k} s, \mathbf{k}_{1} s_{1}}^{\mathrm{int}}(t) \widehat{b}_{\mathbf{k}_{1} s_{1}}(t)\right), \\
& \frac{\mathrm{d} \widehat{b}_{\mathbf{k} s}^{\dagger}(t)}{\mathrm{d} t}=\frac{\mathrm{i}}{\hbar}\left(E_{\mathbf{k} s} \widehat{b}_{\mathbf{k} s}^{\dagger}(t)+\sum_{\mathbf{k}_{1}, s_{1}} H_{\mathbf{k}_{1} s_{1}, \mathbf{k} s}^{\mathrm{int}}(t) \widehat{b}_{\mathbf{k}_{1} s_{1}}^{\dagger}(t)\right) .
\end{aligned}
$$

If one consider electromagnetic wave as a small perturbation, solution for creation (annihilation) operators in zero order approximation has the following form:

$$
\widehat{b}_{\mathbf{k} s}(t)=\widehat{b}_{\mathbf{k} s}^{A} \exp \left(-\frac{\mathrm{i}}{\hbar} E_{\mathbf{k} s} t\right)
$$

where $\widehat{b}_{\mathbf{k} s}^{A}$ can be referred to as an "amplitude" of the annihilation operator, which is independent on time. Then in the first order approximation we can add a time dependence to the amplitude:

$$
\widehat{b}_{\mathbf{k} s}^{A}(t)=\widehat{b}_{\mathbf{k} s}^{(0)}+\widehat{b}_{\mathbf{k} s}^{(1)}(t)
$$

where $\widehat{b}_{\mathbf{k} s}^{(0)}$ is a time independent part of the amplitude, and $\widehat{b}_{\mathbf{k} s}^{(1)}(t)$ is a time dependent one. Substituting Eq. (13) into the Eq. (12) and further into Eq.(10), one can derive

$$
\begin{aligned}
& \frac{\mathrm{d} \widehat{b}_{\mathbf{k} s}^{(1)}(t)}{\mathrm{d} t}=-\frac{\mathrm{i}}{\hbar} \sum_{\mathbf{k}_{1} s_{1}} H_{\mathbf{k} s, \mathbf{k}_{1} s_{1}}^{\mathrm{int}}(t) \widehat{b}_{\mathbf{k}_{1} s_{1}}^{(0)} \exp \left(-\frac{\mathrm{i}}{\hbar}\left(E_{\mathbf{k}_{1} s_{1}}-E_{\mathbf{k} s}\right)\right), \\
& \frac{\mathrm{d} \widehat{b}_{\mathbf{k} s}^{(1) \dagger}(t)}{\mathrm{d} t}=\frac{\mathrm{i}}{\hbar} \sum_{\mathbf{k}_{1} s_{1}} H_{\mathbf{k}_{1} s_{1}, \mathbf{k} s}^{\mathrm{int}}(t) \widehat{b}_{\mathbf{k}_{1} s_{1}}^{(0) \dagger} \exp \left(\frac{\mathrm{i}}{\hbar}\left(E_{\mathbf{k}_{1} s_{1}}-E_{\mathbf{k} s}\right)\right) .
\end{aligned}
$$

Performing the temporal Fourier transform of (14-15) it is produced:

$$
\begin{aligned}
\widehat{b}_{\mathbf{k} s}^{(1)}(\omega) & =\frac{1}{\hbar \omega} \sum_{\mathbf{k}_{1} s_{1}} \widetilde{H}_{\mathbf{k} s, \mathbf{k}_{1} s_{1}}^{\mathrm{int}}\left(\omega-\frac{E_{\mathbf{k}_{1} s_{1}}-E_{\mathbf{k} s}}{\hbar}\right) \widehat{b}_{\mathbf{k}_{1} s_{1}}^{(0)} \\
\widehat{b}_{\mathbf{k} s}^{(1) \dagger}(\omega) & =-\frac{1}{\hbar \omega} \sum_{\mathbf{k}_{1} s_{1}} \widetilde{H}_{\mathbf{k}_{1} s_{1}, \mathbf{k} s}^{\mathrm{int}}\left(\omega+\frac{E_{\mathbf{k}_{1} s_{1}}-E_{\mathbf{k} s}}{\hbar}\right) \widehat{b}_{\mathbf{k}_{1} s_{1}}^{(0) \dagger}
\end{aligned}
$$

where $\widehat{b}_{\mathbf{k} s}^{(1)}(\omega)=\int \widehat{b}_{\mathbf{k} s}^{(1)}(t) \exp (\mathrm{i} \omega t) \mathrm{d} t$ and similarly for time dependent part of the amplitude of creation operator, $\widetilde{H}_{\mathbf{k}_{1} s_{1}, \mathbf{k}_{2} s_{2}}^{\text {int }}(\omega)$ is the temporal Fourier transform of matrix element from Eq.(9):

$$
\widetilde{H}_{\mathbf{k}_{1} s_{1}, \mathbf{k}_{2} s_{2}}^{\text {int }}(\omega)=e \int\left(\int \Phi(\mathbf{r}, t) \exp (\mathrm{i} \omega t) \mathrm{d} t\right) \psi_{s_{1}}\left(\mathbf{r}, \mathbf{k}_{1}\right) \psi_{s_{2}}\left(\mathbf{r}, \mathbf{k}_{2}\right) \mathrm{d} \mathbf{r}
$$


Substitution of Eqs.(12-13) in the Eq.(4) gives for the electron charge density (2):

$$
\rho(\mathbf{r}, t)=\rho_{0}(\mathbf{r})+\rho_{1}(\mathbf{r}, t)
$$

where $\rho_{0}(\mathbf{r})$ is the negative equilibrium charge density completely compensated by positive charges of atom cores, and $\rho_{1}(\mathbf{r}, t)$ is an additional charge density generated by the perturbation. The second one should be substituted in the right hand side of Eq.(1).

Performing Fourier transform on $x, y, t$ it is produced

$$
\begin{aligned}
\widetilde{\rho}_{1}\left(\mathbf{k}_{\|}, z, \omega\right) & =e \sum_{\mathbf{k}_{1} s_{1}} \sum_{\mathbf{k}_{2} s_{2}} \mathcal{F}_{\mathbf{k}_{1} s_{1}}^{\mathbf{k}_{2} s_{2}}\left(\mathbf{k}_{\|}, z\right) \widetilde{H}_{\mathbf{k}_{2} s_{2}, \mathbf{k}_{1} s_{1}}^{\mathrm{int}}(\omega) \mathcal{R}_{\mathbf{k}_{1} s_{1}}^{\mathbf{k}_{2} s_{2}}(\omega), \\
\mathcal{F}_{\mathbf{k}_{1} s_{1}}^{\mathbf{k}_{2} s_{2}}\left(\mathbf{k}_{\|}, z\right) & =\int \exp \left(-\mathrm{i} \mathbf{k}_{\|} \mathbf{r}_{\|}\right) \psi_{s_{1}}^{*}\left(\mathbf{r}, \mathbf{k}_{1}\right) \psi_{s_{2}}\left(\mathbf{r}, \mathbf{k}_{2}\right) \mathrm{d} \mathbf{r}_{\|}, \\
\mathcal{R}_{\mathbf{k}_{1} s_{1}}^{\mathbf{k}_{2} s_{2}}(\omega) & =\frac{\left(n_{\mathbf{k}_{1} s_{1}}-n_{\mathbf{k}_{2} s_{2}}\right)}{\hbar \omega+E_{\mathbf{k}_{1} s_{1}}-E_{\mathbf{k}_{2} s_{2}}}
\end{aligned}
$$

in the equations above $\mathbf{k}_{\|}=\left(k_{x}, k_{y}\right)$ is the component of wave vector along graphene surface, $\mathbf{r}_{\|}=(x, y)$ is a radius vector, laying in the graphene plane, $n_{\mathbf{k}, s}=\left\langle 0\left|\widehat{b}_{\mathbf{k} s}^{(0) \dagger} \widehat{b}_{\mathbf{k} s}^{(0)}\right| 0\right\rangle$ is the occupation number of the state $\mathbf{k}, s$. Fourier transformation applied to the left hand side of Eq.(1) gives

$$
\left(-\frac{\omega^{2}}{c^{2}}+k_{\|}^{2}-\frac{\partial^{2}}{\partial z^{2}}\right) \widetilde{\Phi}\left(\mathbf{k}_{\|}, z, \omega\right)
$$

where $k_{\|}=\left|\mathbf{k}_{\|}\right|$, and $\widetilde{\Phi}\left(\mathbf{k}_{\|}, z, \omega\right)$ is the Fourier transform of the scalar potential $\Phi(\mathbf{r}, t)$ :

$$
\widetilde{\Phi}\left(\mathbf{k}_{\|}, z, \omega\right)=\iint \Phi(\mathbf{r}, t) \exp \left(\mathrm{i}\left(\omega t-\mathbf{k}_{\|} \mathbf{r}_{\|}\right)\right) \mathrm{d} \mathbf{r}_{\|} \mathrm{d} t
$$

However, for a slowed down electromagnetic waves the following inequality is fulfilled: $\frac{\omega}{c} \ll k_{\|}$. That is why (23) can be replaced by

$$
\left(k_{\|}^{2}-\frac{\partial^{2}}{\partial z^{2}}\right) \widetilde{\Phi}\left(\mathbf{k}_{\|}, z, \omega\right)
$$

Finally we have the following equation :

$$
\left(k_{\|}^{2}-\frac{\partial^{2}}{\partial z^{2}}\right) \widetilde{\Phi}\left(\mathbf{k}_{\|}, z, \omega\right)=4 \pi e \sum_{\mathbf{k}_{1} s_{1}} \sum_{\mathbf{k}_{2} s_{2}} \mathcal{F}_{\mathbf{k}_{1} s_{1}}^{\mathbf{k}_{2} s_{2}}\left(\mathbf{k}_{\|}, z\right) \widetilde{H}_{\mathbf{k}_{2} s_{2}, \mathbf{k}_{1} s_{1}}^{\mathrm{int}}(\omega) \mathcal{R}_{\mathbf{k}_{1} s_{1}}^{\mathbf{k}_{2} s_{2}}(\omega) .
$$




\section{Self-consistent problem solution}

In the previous section the basic equation (26) for the self-consistent problem was derived. For analysing the graphene system with the help of this equation, the wave functions $\psi_{s}(\mathbf{r}, \mathbf{k})$ and the energies of electron states $E_{\mathbf{k}, s}$ should be defined. Tightbinding method can be used to determine these quantities. Within a framework of this method the wave functions are linear combinations of the Bloch functions:

$$
\psi_{s}(\mathbf{r}, \mathbf{k})=\sum_{i=1}^{\sigma} C_{i s}(\mathbf{k}) \psi_{i}^{(\mathrm{B})}(\mathbf{r}, \mathbf{k}),
$$

where $\sigma$ is a number of carbon atoms in the unit cell of the $n$-layer graphene system, $C_{i, s}$ are the unknown coefficients to be found and $\psi_{i}^{(B)}(\mathbf{r}, \mathbf{k})$ are the Bloch functions:

$$
\psi_{i}^{(\mathrm{B})}(\mathbf{r}, \mathbf{k})=\frac{1}{\sqrt{N}} \sum_{j=1}^{N} \phi_{i}\left(\mathbf{r}-\mathbf{r}_{i j}\right) \exp \left(\mathrm{ikr}_{i j}\right)
$$

where $N$ is a number of unit cells in the system, $\mathbf{r}_{i j}$ is the radius vector that points out position of $i$-th atom in the $j$-th unit cell, $\phi_{i}(\mathbf{r})$ is $\pi$-electron atomic orbital.

The unknown coefficients $C_{i s}(\mathbf{k})$ are determined by solving eigenproblem for the n-layer graphene system:

$$
M C_{s}=0,
$$

where $M$ is a $\sigma \times \sigma$ matrix, and $C_{s}=\left(C_{1 s}(\mathbf{k}), \ldots, C_{\sigma s}(\mathbf{k})\right)^{\mathrm{T}}$ is a column vector. For the case of graphene bilayer the matrix $M$ has the form

$$
\left(\begin{array}{cccc}
E_{0}+\Delta-E_{\mathbf{k}} & \gamma_{0} f\left(k_{x}, k_{y}\right) & \gamma_{1} & \gamma_{4} f^{*}\left(k_{x}, k_{y}\right) \\
\gamma_{0} f^{*}\left(k_{x}, k_{y}\right) & E_{0}-E_{\mathbf{k}} & \gamma_{4} f^{*}\left(k_{x}, k_{y}\right) & \gamma_{3} f\left(k_{x}, k_{y}\right) \\
\gamma_{1} & \gamma_{4} f\left(k_{x}, k_{y}\right) & E_{0}+\Delta-E_{\mathbf{k}} & \gamma_{0} f^{*}\left(k_{x}, k_{y}\right) \\
\gamma_{4} f\left(k_{x}, k_{y}\right) & \gamma_{3} f^{*}\left(k_{x}, k_{y}\right) & \gamma_{0} f\left(k_{x}, k_{y}\right) & E_{0}-E_{\mathbf{k}}
\end{array}\right)
$$

and for graphene single layer

$$
\left(\begin{array}{cc}
E_{0}+\Delta-E_{\mathbf{k}} & \gamma_{0} f\left(k_{x}, k_{y}\right) \\
\gamma_{0} f^{*}\left(k_{x}, k_{y}\right) & E_{0}-E_{\mathbf{k}}
\end{array}\right)
$$

where $E_{0}, \Delta, \gamma_{i}$ are the tight-binding model parameters taken from [17]. Function $f\left(k_{x}, k_{y}\right)$ has the form

$$
f\left(k_{x}, k_{y}\right)=\exp \left(\frac{\mathrm{i} k_{x} a}{\sqrt{3}}\right)+2 \exp \left(\frac{\mathrm{i} k_{x} a}{2 \sqrt{3}} \cos \left(\frac{k_{y} a}{2}\right)\right),
$$

where $k_{x}, k_{y}$ are projections of the momentum onto $x$ and $y$ axis, respectively, $a$ is modulus of vectors $\mathbf{a}_{1}$ and $\mathbf{a}_{2}$, see Fig. 1 .

In general, the eigenproblem (29) has nonzero solution if determinant of its matrix is equal to zero: 


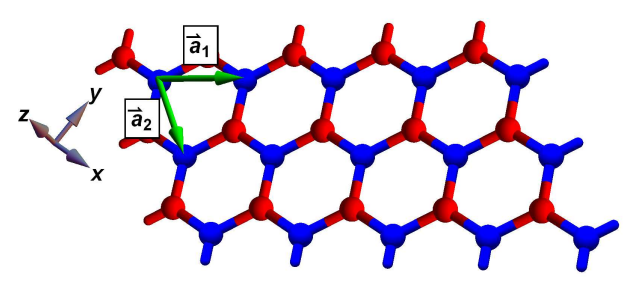

Fig. 1 Graphene single layer crystal lattice. Vectors $\mathbf{a}_{1}, \mathbf{a}_{2}$ are the basis vectors of the lattice. Carbon atoms inequivalent due to symmetry of the lattice are depicted with different colours (red and blue).

$$
\operatorname{det} M=0 \text {. }
$$

Equation (33) is the polynomial equation of the $\sigma$-th order, and it has $\sigma$ roots for each value of $\mathbf{k}$. These solutions can be numbered by index $s$ varing from 1 to $\sigma$.

Assuming smallness of perturbed part of electron density, the all terms in the left hand side of the Eq.(26) can be written in the form:

$$
\int g_{\mathbf{k}_{\|}, \mathbf{r}_{\|}} \phi_{i}^{*}\left(\mathbf{r}-\mathbf{r}_{i j}\right) \phi_{m}\left(\mathbf{r}-\mathbf{r}_{m n}\right) \mathrm{d} \mathbf{r}_{\|}=\delta_{i m, j n} \int g_{\mathbf{k}_{\|}, \mathbf{r}_{\|}}\left|\phi_{i}\left(\mathbf{r}-\mathbf{r}_{i j}\right)\right|^{2} \mathrm{~d} \mathbf{r}_{\|},
$$

where $g_{\mathbf{k}_{\|}, \mathbf{r}_{\|}}$is an arbitrary bounded function of arguments $\mathbf{k}_{\|}$and $\mathbf{r}, \delta_{i m, j n}$ is the Kronecker delta. Using (34), one can transform (21) to the following form:

$$
\mathcal{F}_{\mathbf{k}_{1} s_{1}}^{\mathbf{k}_{2} s_{2}}\left(\mathbf{k}_{\|}, z\right)=\sum_{i=1}^{\sigma} C_{i s_{1}}^{*}\left(\mathbf{k}_{1}\right) C_{i s_{2}}\left(\mathbf{k}_{2}\right) F_{i}\left(\mathbf{k}_{\|}, z\right) \delta_{\mathbf{k}_{\|}, \mathbf{k}_{2}-\mathbf{k}_{1}}
$$

where $\delta_{\mathbf{k}_{\|}, \mathbf{k}_{2}-\mathbf{k}_{1}}$ is a Kroneker delta that provides the quasimomentum conservation during electron-photon interaction, $F_{i}\left(\mathbf{k}_{\|}, z\right)$ is a function that can be referred to as the atomic orbital form factor having the next form:

$$
F_{i}\left(\mathbf{k}_{\|}, z\right)=\int \exp \left(-\mathrm{i} \mathbf{k}_{\|} \mathbf{r}_{\|}\right)\left|\phi_{i}\left(\mathbf{r}_{\|}, z-z_{i}\right)\right|^{2} \mathrm{~d} \mathbf{r}_{\|} .
$$

Again using the tight-binding condition (34) and additionally Eqs.(27-28), one can present matrix elements (18) as follows:

$$
\widetilde{H}_{\mathbf{k}_{1} s_{1}, \mathbf{k}_{2} s_{2}}^{\text {int }}(\omega)=\sum_{i=1}^{\sigma} C_{i s_{2}}^{*}\left(\mathbf{k}_{2}\right) C_{i s_{1}}\left(\mathbf{k}_{1}\right) A_{i}\left(\mathbf{k}_{2}-\mathbf{k}_{1}, \omega\right),
$$

where 


$$
A_{i}\left(\mathbf{k}_{2}-\mathbf{k}_{1}, \omega\right)=\int F_{i}\left(\mathbf{k}_{1}-\mathbf{k}_{2}, z\right) \frac{e}{S} \widetilde{\Phi}\left(\mathbf{k}_{2}-\mathbf{k}_{1}, z, \omega\right) \mathrm{d} z
$$

here $S$ is a square of the $n$-layer graphene sheet.

Substitution of Eqs.(37,35) into the Eq.(26) gives

$$
\left(k_{\|}^{2}-\frac{\partial^{2}}{\partial z^{2}}\right) \widetilde{\Phi}\left(\mathbf{k}_{\|}, z, \omega\right)=\sum_{i=1}^{\sigma} f_{i}\left(\mathbf{k}_{\|}, z, \omega\right) A_{i}\left(\mathbf{k}_{\|}, \omega\right)
$$

where

$$
f_{i}\left(\mathbf{k}_{\|}, z, \omega\right)=4 \pi e \sum_{\mathbf{k}_{1} s_{1}, \mathbf{k}_{2} s_{2}} \mathcal{D}_{\mathbf{k}_{1} s_{1}, i}^{\mathbf{k}_{2} s_{2}}\left(\mathbf{k}_{\|}, z\right) \delta_{\mathbf{k}_{\|}, \mathbf{k}_{2}-\mathbf{k}_{1}} \mathcal{R}_{\mathbf{k}_{1} s_{1}}^{\mathbf{k}_{2} s_{2}}(\omega)
$$

and

$$
\mathcal{D}_{\mathbf{k}_{1} s_{1}, i}^{\mathbf{k}_{2} s_{2}}\left(\mathbf{k}_{\|}, z\right)=\sum_{m=1}^{\sigma} C_{m s_{1}}^{*}\left(\mathbf{k}_{1}\right) C_{m s_{2}}\left(\mathbf{k}_{2}\right) C_{i s_{2}}^{*}\left(\mathbf{k}_{2}\right) C_{i s_{1}}\left(\mathbf{k}_{1}\right) F_{i}\left(\mathbf{k}_{\|}, z\right) .
$$

In the long wave approximation inequality $k_{\|} a \ll 1$ is fulfilled. Therefore the approximation $\exp \left(-\mathrm{i} \mathbf{k}_{\|} \mathbf{r}_{\|}\right) \rightarrow 1$ in the expressions for atomic form factor can be used. We shall use approximation $\int\left|\phi_{i}\left(\mathbf{r}_{\|}, z-z_{i}\right)\right|^{2} \mathrm{~d} \mathbf{r}_{\|} \rightarrow \delta\left(z-z_{i}\right)$ which corresponds to the electron wave functions concentrated on graphene monolayers. Applying these approximations one can reduce Eq.(38) to the form

$$
A_{i}\left(\mathbf{k}_{\|}, \omega\right)=\frac{e}{S} \widetilde{\Phi}\left(\mathbf{k}_{\|}, z_{i}, \omega\right)
$$

where the quasimomentum conservation law have been used. Smallness of the photon momentum leads to possibility of the following replacement:

$$
\mathcal{D}_{\mathbf{k}_{1} s_{1}, i}^{\mathbf{k}_{2} s_{2}}\left(\mathbf{k}_{\|}, z\right) \rightarrow \mathcal{D}_{s_{1}, i}^{\prime s_{2}}(z)
$$

where

$$
\mathcal{D}_{s_{1}, i}^{\prime s_{2}}(z)=\sum_{m=1}^{\sigma} \delta_{s_{1}, s_{2}}\left|C_{m s_{1}}\right|^{2}\left|C_{i s_{1}}\right|^{2} \delta\left(z-z_{i}\right),
$$

$\delta_{s_{1}, s_{2}}$ is the Kronecker delta arisen from the consideration of the eigenproblem (29), also note that the absolute value of the coefficients $C_{i j}(\mathbf{k})$ does not depend on $\mathbf{k}$, $\delta\left(z-z_{i}\right)$ is the Dirac delta corresponding to the new form of the atomic orbital form factor.

Applying the replacement rule (43) to the Eq.(40) and taking into account Eq.(42), one can obtain the new form of (39):

$$
\left(k_{\|}^{2}-\frac{\partial^{2}}{\partial z^{2}}\right) \widetilde{\Phi}\left(\mathbf{k}_{\|}, z, \omega\right)=\frac{4 \pi e^{2}}{S} \sum_{\mathbf{k}_{1} s_{1}, s_{2}} \mathcal{R}_{\mathbf{k}_{1} s_{1}}^{\mathbf{k}_{1}+\mathbf{k}_{\|}, s_{2}}(\omega) \mathcal{P}_{s_{1}}^{s_{2}}\left(\mathbf{k}_{\|}, z, \omega\right),
$$

where

$$
\mathcal{P}_{s_{1}}^{s_{2}}\left(\mathbf{k}_{\|}, z, \omega\right)=\sum_{i=1}^{\sigma} \mathcal{D}_{s_{1}, i}^{\prime s_{2}}(z) \widetilde{\Phi}\left(\mathbf{k}_{\|}, z_{i}, \omega\right)
$$


For equation (45), the following boundary conditions can be stated:

$$
\begin{aligned}
\left.\widetilde{\Phi}\right|_{z \rightarrow \pm \infty} & =0, \\
\left.\widetilde{\Phi}\right|_{z=z_{i}^{(1)}-0} & =\left.\widetilde{\Phi}\right|_{z=z_{i}^{(1)}+0}, \\
\left.\frac{\mathrm{d} \widetilde{\Phi}}{\mathrm{d} z}\right|_{z=z_{i}^{(1)}-0}-\left.\frac{\mathrm{d} \widetilde{\Phi}}{\mathrm{d} z}\right|_{z=z_{i}^{(1)}+0} & =\frac{4 \pi e^{2}}{S} \sum_{s_{1}} \mathcal{P}_{s_{1}}^{\prime}\left(\mathbf{k}_{\|}, \omega\right) \sum_{\mathbf{k}_{1}} \mathcal{R}_{\mathbf{k}_{1} s_{1}}^{\mathbf{k}_{1}+\mathbf{k}_{\|}, s_{1}}(\omega),
\end{aligned}
$$

where

$$
\mathcal{P}_{s_{1}}^{\prime}\left(\mathbf{k}_{\|}, \omega\right)=\sum_{m, i=1}^{\sigma}\left|C_{m s_{1}}\right|^{2}\left|C_{i s_{1}}\right|^{2} \widetilde{\Phi}\left(\mathbf{k}_{\|}, z_{i}, \omega\right) .
$$

Boundary condition (47) means finiteness of the scalar potential throughout the space. Conditions (48) and (49) correspond to the continuity of the scalar potential and the discontinuity of electric field strength on each sheet of the $n$-layer graphene system, respectively. The third boundary condition (49) was obtained by integration of Eq.(45) in the vicinity of each graphene plane.

For the regions $z \neq z_{i}$ solution of the Eq.(45) has the next form

$$
\widetilde{\Phi}\left(k_{\|}, z, \omega\right)=\sum_{i=1}^{n} c_{1, i}\left(k_{\|} \omega\right) \exp \left[k_{\|}\left(z-z_{i}^{(1)}\right)\right]+c_{2, i}\left(k_{\|} \omega\right) \exp \left[-k_{\|}\left(z-z_{i}^{(1)}\right)\right]
$$

where $n$ is the number of layers, $z_{i}^{(1)}$ is the position of the $i$-th layer, $c_{j, i}\left(k_{\|} \omega\right)$ are coefficients to be defined. A homogeneous system of algebraic equations for $c_{j, i}\left(k_{\|} \omega\right)$ can be obtained if one apply the boundary conditions (47-49) to the solution specified above. Such system has nonzero solution when determinant of its matrix equal to zero. This equality gives the dispersion equation and dependence of wave frequency on the wave vector. Further it will be reduced to two particular cases: graphene single layer and bilayer.

\section{Dispersion equations for graphene single layer and bilayer}

Dispersion equation for the $n$-layer graphene system derived in the way described in the previous section is too cumbersome to analyse in a general form. Its complexity strongly increase with the number of graphene layers in the system. From practical point of view two cases have huge attraction. They are graphene single layer and bilayer. The former is interesting because of linear dispersion of electrons [16], the latter - due to controllable energy gap in the band structure [15]. Besides, the second case is interesting, because of opportunity to investigate the dependence of electromagnetic wave dispersion on interaction between adjacent layers.

Let begin analysis from the more simple case of graphene single layer. We consider doped graphene with injected electron in the conduction band and take into 
account transition in conduction band only. Low temperature limit is considered in what follows. From mathematical point of view it results in the step function form of the Fermi-Dirac distribution describing the average occupation numbers $n_{\mathbf{k}, s}$. Moreover, long wave approximation is applied again in calculations. This time it means that the denominator of the resonance factor should be expand to first order terms with respect to $\mathbf{k}_{\|}$. Producing that, one can obtain the following dispersion equation for the graphene single layer:

$$
\frac{4 \pi^{2} e^{2}}{\hbar v_{F}} k_{F} \frac{\omega-\left(\omega^{2}-v_{F}^{2} k^{2}\right)^{1 / 2}}{k\left(\omega^{2}-v_{F}^{2} k^{2}\right)^{1 / 2}}=1
$$

where $k_{F}$ is the momentum of an electron on the Fermi surface in the graphene single layer. Dependence of electromagnetic wave frequency on wave vector is shown on the Fig. 2. Dependence of the plasmon phase speed on wave vector is presented on

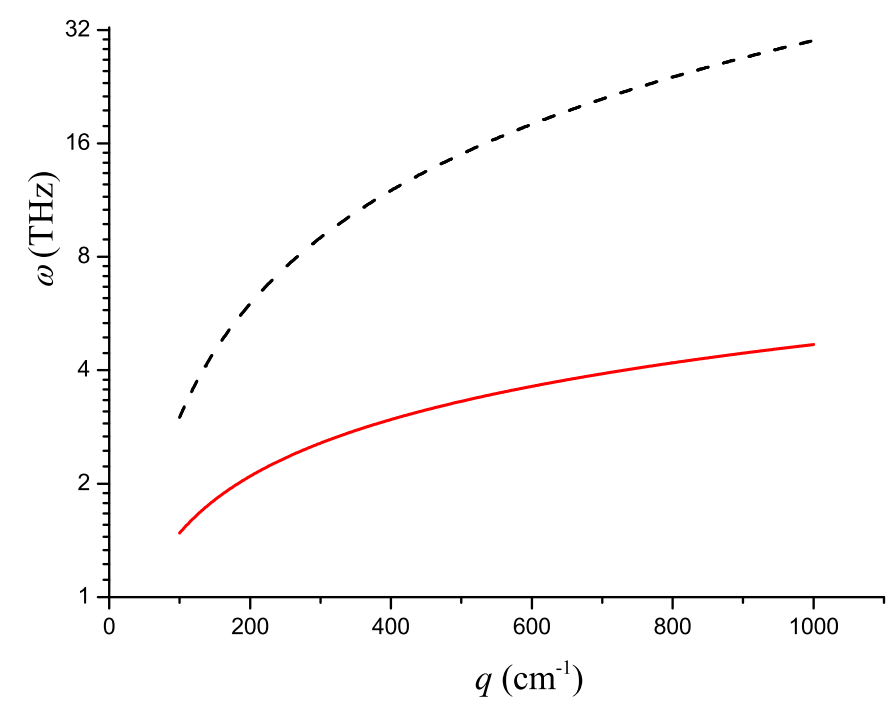

Fig. 2 The dependence of plasmon frequency $(\mathrm{THz})$ on wave vector $\left(\mathrm{cm}^{-1}\right)$. Density of doped electrons is taken to be $10^{12} \mathrm{~cm}^{-2}$. Dashed curve shows the light cone (logarithmic scale is used).

the Fig.(3). It can be seen that plasmon phase speed in graphene single layer is 3-6 times smaller than the speed of light in the vacuum and it increase with the density of doped electrons.

Another case of our interest is the graphene bilayer. Using the same assumptions as for the graphene single layer, we obtain the following dispersion equation: 


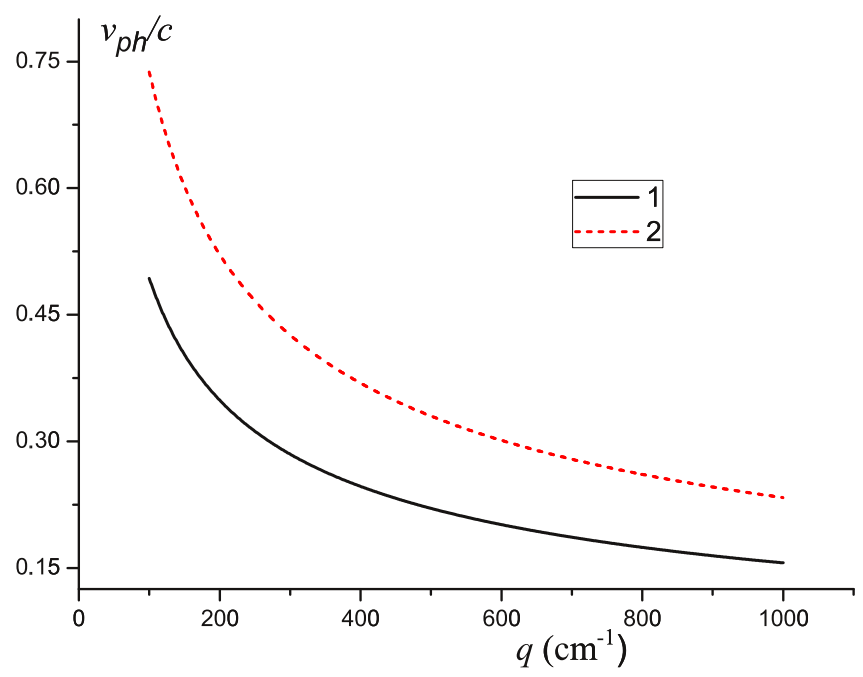

Fig. 3 The dependence of plasmon phase speed $(1 / c$, where $c$ is the speed of light in the vacuum) on wave vector $\left(\mathrm{cm}^{-1}\right)$. The curves (1) and (2) are plotted for densities of doped electrons $10^{12} \mathrm{~cm}^{-2}$ and $5 \times 10^{-12} \mathrm{~cm}^{-2}$, respectively.

$$
\left(1-\frac{2 \pi e}{k} \Pi_{1}\right)\left(1-\frac{2 \pi e}{k} \Pi_{2}\right)-\frac{4 \pi^{2} e^{2}}{k^{2}} \Pi_{1} \Pi_{2} \exp (-2 k d)=0 .
$$

where $d$ is the distance between graphene sheets in graphene bilayer, $\Pi_{1,2}$ is referred to as irreducible polarizability, having the form

$$
\Pi_{i}=\sum_{k_{1}<k_{F}}\left(\frac{1}{\hbar \omega+E_{\mathbf{k}_{1}-\mathbf{k}, i}-E_{\mathbf{k}_{1}, i}}-\frac{1}{\hbar \omega+E_{\mathbf{k}_{1}, i}-E_{\mathbf{k}_{1}+\mathbf{k}, i}}\right),
$$

There are two irreducible polatizabilities corresponding to two different conduction bands.

In long wave approximation irreducible polarizability can be presented in the form $\Pi_{i}=\Gamma_{i} / \omega^{2}$, where $\Gamma_{i}$ depends on $\mathbf{k}$ and does not depend on the frequency $\omega$. Solution for the frequency has very simple analytical form in that case:

$$
\omega_{1,2}^{2}(k)=\frac{\Gamma_{1}+\Gamma_{2}}{2} \pm \sqrt{\left(\frac{\Gamma_{1}+\Gamma_{2}}{2}\right)^{2}-\Gamma_{1} \Gamma_{2}[1-\exp (-2 k d)]},
$$

The sing "-" in the solution (55) correspond to the acoustic plasmon mode, having phase speed considerably smaller than plasmons in graphenen single layer. Let us remind that this solution is obtained within the assumption that electron wave functions concentrated on graphene layers. Such situation should be observed for spatially separated two graphene monolayers. In this system the distance between 
layers can be turned. As a result the plasmon frequency and phase speed can also be controlled, that is clearly seen on Fig. (4).

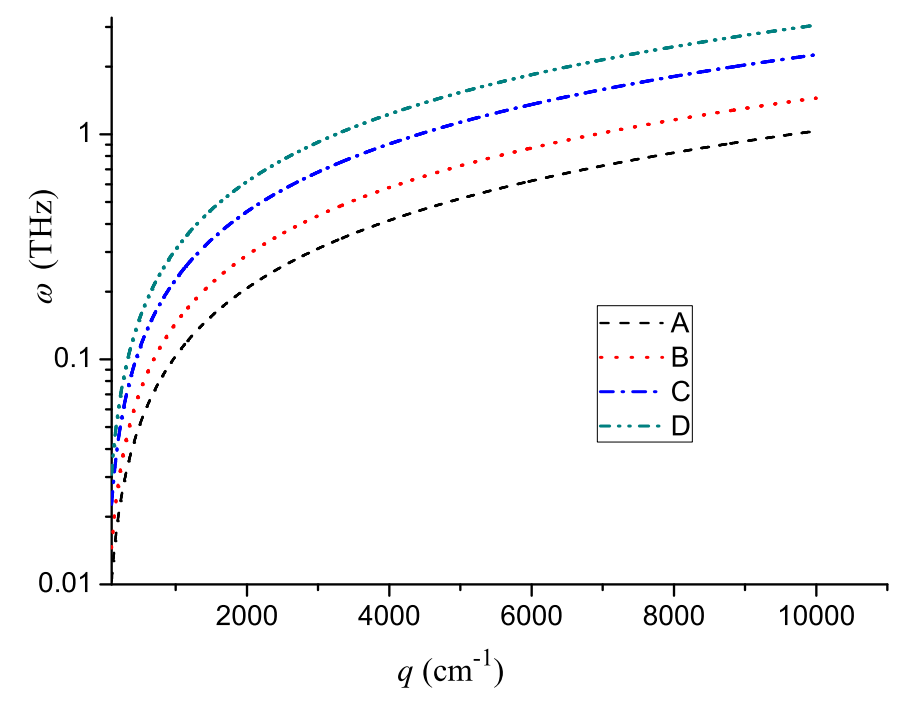

Fig. 4 The dependence of the plasmon frequency $\omega$ on the wave number $q$ for two spatially expanded graphene monolayers: the distance $d$ between layers is (A) $4 \mathrm{~nm}$, (B) $30 \mathrm{~nm}$, (C) $100 \mathrm{~nm}$, (D) $200 \mathrm{~nm}$ for doping electron densities $n_{1}=n_{2}=10^{12} \mathrm{~cm}^{-2}$.

\section{Conclusions}

General method of obtaining dispersion equations for surface plasmons in the $n$ layer graphene system was developed. This method along with long wave and tightbinding approximations was applied to derive dispersion equations for graphene single layer and bilayer. It was shown that in the case of single layer graphene the phase speed of plasmon is only 3-6 times smaller than the speed of light in the vacuum, that is not enough for Čerenkov synchronization with $\pi$ electrons. Meanwhile, graphene bilayer demonstrates desired possibility of phase speed retardation up to the Fermi velocity of $\pi$-electrons in graphene. That paves the way to the synchronization with non-relativistic $\pi$-electrons, e.g. inner currents of the graphene bilayer. Thus, graphene bilayer seems to be challenging material for nanoscale Cerenkovtype emitters in the terahertz region. 


\section{References}

1. Barnes, W.L., Ebbesen, T.W.: Surface plasmon subwavelength optics. Nature 424, 824-830 (2003)

2. Anker, J.N., Ebbesen, T.W.: Biosensing with plasmonic nanosensors. Nature Matter 7, 442$453(2008)$

3. Liu, Y., Bartal, G., Genov, D.A., Zhang, X.: Subwavelength descrete solitons in nonlinear metamaterials. Phys. Rev. Lett. 99, 153901 (2007)

4. Stockman, M.I.: Nanofocusing of optical energy in tapered palsmonic waveguides. Phys. Rev. Lett. 93, 137404 (2004)

5. Ren-Min, M., Oulton, R.F., Volker, Sorger, J., Guy, B. and Zhang, X. : Room-temperature sub-diffraction-limited plasmon laser by total internal reflection. Nature Materials 10, 110$113(2011)$

6. Batrakov, K. G., Kuzhir, P. P., Maksimenko, S. A.: Radiative instability of electron beam in carbon nanotubes. Proc. SPIE 6328, 63280Z (2006)

7. Batrakov, K.G., Maksimenko, S.A., Kuzhir, P.P., Thomsem, C.: Carbon nanotube as a Cherenkov-type light emitter and free electron laser. Phys. Rev. B. 70, 125408-125420 (2009)

8. Batrakov, K.G., Kuzhir, P.P., and Maksimenko, S.A.: Toward the nano-FEL: undulator and Cherenkov mechanisms of light emission in carbon nanotubes. Physica E 40, 1065-1068 (2008)

9. Batrakov, K. G., Kuzhir, P. P., and Maksimenko, S. A.: Stimulated emission of electron beam in nanotube bundles. Physica E 40, 2370-2374 (2008)

10. Slepyan, G.Ya., Maksimenko, S.A., Lakhtakia, A., Yevtushenko, O., Gusakov, A.V.: Electrodynamics of carbon nanotubes: Dynamic conductivity, impedance boundary conditions and surface wave propagation. Phys. Rev. B. 60, 17136-17149 (1999)

11. Frank, S., Poncharal, P., Wang, Z.L., de Heer, W.A. : Carbon Nanotube Quantum Resistors. Science 280, 1744 (1998)

12. Berger, C., Yi, Y., Wang, Z.L., de Heer, W.A.: Multiwalled carbon nanotubes are ballistic conductors at room temperature. Appl. Phys. A 74, 363-365 (2002)

13. Wei, B.Q., Vajtai, R., Ajayan, P.M.: Reliability and current carrying capacity of carbon nanotubes. Appl. Phys. Lett. 79, 1172 (2001)

14. Batrakov, K. G., Kuzhir, P. P., Maksimenko, S. A.: Cherenkov synchronism: Non-relativistic electron beam in multi-walled carbon nanotube and multi-layer graphene. Physica B: Physics of Condensed Matter 405, 3050-3053 (2010)

15. Zhang, Y., Tang, T., Girit, C., Hao, Z., Martin, M.C., Zettl, A., Crommie, M.F., Shen, Y.R., and Wang, F.: Direct observation of a widely tunable bandgap in bilayer graphene. Nature 459, 820-823 (2009)

16. Saito, R., Dresselhaus, G., Dresselhaus, M.: Physical Properties of Carbon Nanotubes. Imperial College Press, London (1998)

17. Partoens, B., Peeters, F.M.: From graphene to graphite: Electronic structure around the K points. Phys. Rev. B 74, 075404 (2006) 